\title{
The influence of duloxetine on detrusor overactivity in rats with depression induced by 13-cis-retinoic acid
}

\author{
Andrzej Wróbel $^{1}$ (D) - Ewa Rechberger ${ }^{1} \cdot$ Tomasz Rechberger $^{1}$
}

Received: 21 March 2017 / Accepted: 30 June 2017 / Published online: 31 July 2017

(C) The Author(s) 2017. This article is an open access publication

\begin{abstract}
Introduction and hypothesis The aim of this study was to assess the efficacy of duloxetine in an animal model of detrusor overactivity induced by depression.

Methods After 6 weeks of 13-cis-retinoic acid administration at a dose of $1 \mathrm{mg} / \mathrm{kg} / \mathrm{day}$, rats were given duloxetine at a dose of $1 \mathrm{mg} / \mathrm{kg}$. This was followed by conscious cystometry, a forced swim test, and locomotor activity measurement. The levels of corticotropin-releasing factor (CRF) in the hypothalamus, amygdala and plasma were also determined.

Results Duloxetine treatment led to a reduction in detrusor overactivity symptoms induced by the retinoid. Decreases were observed in cystometric parameters including the detrusor overactivity index, and the amplitude and frequency of nonvoiding contractions, while increases were seen in bladder compliance and the volume threshold to elicit nonvoiding contractions. No statistically significant differences were found in basal pressure, threshold pressure, micturition voiding pressure, postvoid residual, volume threshold, voiding efficiency, intercontraction interval, bladder contraction duration or relaxation time. Duloxetine also reduced the immobility time to that observed in control animals, while it did not affect locomotor activity. Its effects also included lowering of the CRF levels in the hypothalamus, amygdala and plasma, which increased following the prior administration of the retinoid. The plasma level of 13-cis-retinoic acid in rats corresponded to the levels found in humans.
\end{abstract}

Andrzej Wróbel

wrobelandrzej@yahoo.com

1 Second Department of Gynecology, Medical University of Lublin, Jaczewskiego 8, PL 20-090 Lublin, Poland
Conclusions This is the first study showing the efficacy of duloxetine in an animal model of detrusor overactivity induced by depression. Further studies in patients with detrusor overactivity and coexisting depression are warranted to confirm these experimental results.

Keywords Duloxetine $\cdot$ Detrusor overactivity $\cdot$ Depression Cystometry $\cdot$ Rats

\section{Introduction}

Patients with various clinical types of urinary incontinence are frequently diagnosed with mental disorders including depression. Although the putative link between such urinary tract malfunction was first described in 1964 [1], probably the first to speculate on common neurological pathways for depression and detrusor overactivity (DO) leading to both wet and dry overactive bladder $(\mathrm{OAB})$ were Steers and Lee [2]. Indeed some data suggest that depression might be associated with $\mathrm{OAB}$ syndrome [3], but very few studies have focused on $\mathrm{OAB}$ patients with clinically confirmed psychiatric disorders $[4,5]$. Epidemiological studies have indicated that such a link may exist. It has been demonstrated that patients with $\mathrm{OAB}$ have significantly worse results on the Centre of Epidemiological Studies Depression (CES-D) and quality of sleep scales [6], as well as on the Short Form Health Survey (SF-36) quality of life scale. A positive correlation has been found between depression and the development of the first symptoms of OAB. It has been suggested that depression, as assessed with the Geriatric Depression scale, is a risk factor for $\mathrm{OAB}$ [7]. Of patients diagnosed with depression, $13 \%$ also had symptoms of stress urinary incontinence (SUI) and $42 \%$ had symptoms of OAB or mixed urinary incontinence (MUI), and as many as $60 \%$ of patients with idiopathic urge 
incontinence had a history of depression or a Beck's Depression Inventory score of $>12$.

Corticotropin-releasing factor (CRF) is considered a primary initiator of changes leading to depression. Its level increases especially in the paraventricular nucleus of the hypothalamus, the central nucleus of the amygdala, and Barrington's nucleus, the pontine micturition centre. It displays strong psychotropic properties, predominantly as depressive reactions. Preclinical studies have revealed that $\mathrm{CRF}$ induces depressive reactions by increasing the immobility time in forced swim tests. The $\mathrm{CRF}_{1}$ receptor antagonists show, in turn, antidepressant properties [8]. In forced swim tests the reduction in passive (immobility) behaviour is interpreted as an antidepressantlike effect [9]. Clinical trials have confirmed that patients with depression have significantly increased CRF levels in their cerebrospinal fluid. Post-mortem examinations have also revealed elevated CRF concentrations in the hypothalamus and an increase in the number of CRF-releasing neurons located in the paraventricular nucleus of the hypothalamus.

The effects of $\mathrm{CRF}$ are mediated by $\mathrm{CRF}_{1}$ and $\mathrm{CRF}_{2}$ receptors. Brain regions characterized by high concentrations of these receptors, such as Barrington's nucleus in the brainstem amygdala, the prefrontal cortex and the hippocampus, play an important role, not only in the aetiopathogenesis of depression but also in voiding control. The neurons of Barrington's nucleus, which project to the bladder motoneurons, are considered the switch that initiates the descending limb of the micturition reflex. There is some evidence suggesting that high CRF expression of the neurons of Barrington's nucleus is an important factor regulating bladder detrusor function. It has been found that CRF lowers the threshold of the afferent impulsation of the voiding reflex and increases the contractile activity of the detrusor muscle, whose levels increase in response to bladder distension. In vivo cystometric tests have confirmed the excitatory effects of $\mathrm{CRF}$ on micturition [10]. $\mathrm{CRF}_{1}$-selective antagonists have been found to have a reducing effect on DO. CRF has an effect on the micturition reflex, including at the level of the spinal cord. It has been shown that capsaicin, by selectively destroying afferent $\mathrm{C}$-fibres, induces a decrease in CRF expression, confirming that this neurohormone is vital in the conduction of afferent impulsation from the bladder [11].

Duloxetine is a dual reuptake inhibitor of serotonin and norepinephrine (SNRI) without considerable affinity for these neurotransmitter receptors, and has been approved for the treatment of major depression and SUI. Noradrenaline and serotonin terminals are widespread in brain structures responsible for the body's response to stress, especially in the limbic system. Deficiency in these neurotransmitters induces the development of depression, which is an indication for the administration of SNRIs such as duloxetine in patients with clinical depression [12].

It has been demonstrated that duloxetine increases bladder capacity by increasing urethral sphincter muscle activity without affecting the micturition phase [13]. By inducing an increase in serotonin and norepinephrine concentrations, it modulates the activity of the motoneurons of Onuf's nucleus, and in this way prevents accidental bladder voiding. Only a few sources suggest that duloxetine can inhibit DO development and modulate sensory processing, which would justify its use in $\mathrm{OAB}$ treatment [14-16]; however, the treatment of patients with OAB with this SNRI has not yet been indicated. At present there are no data on the use of duloxetine in the treatment of patients with depression and accompanying $\mathrm{OAB}$ symptoms.

These observations taken together were the basis for the experiments presented here, which had the aim of assessing the effectiveness of duloxetine in an animal model of DO induced by depression [17]. Furthermore, the effects of this SNRI on CRF levels in plasma and brain structures that play a crucial role in both the aetiopathogenesis of depression and the occurrence of $\mathrm{OAB}$ were also assessed.

\section{Materials and methods}

\section{Animals}

All procedures were conducted in accordance with the European Communities Council Directive of 22 September $2010(2010 / 63 /$ EU) and were approved by the Ethics Committee of the Medical University of Lublin.

A total of 60 female Wistar rats were used and randomly assigned to one of the following four treatment groups (15 animals per group):

1. Control

2. Duloxetine $(1 \mathrm{mg} / \mathrm{kg})$

3. 13-cis-Retinoic acid (13-cis-RA; $1 \mathrm{mg} / \mathrm{kg} /$ day)

4. 13-cis-RA plus duloxetine

Rats were experimentally naive and tested once. The animals were weighed at intervals of 1 week until completion of the experiment.

\section{Drugs}

The following drugs were used:

- 13-cis-RA (Tocris Bioscience, Bristol, UK) was dissolved in the dark in a mixture of dimethyl sulphoxide and physiological saline at a ratio of 1:1 just before administration. The solution was injected intraperitoneally at a dose of $1 \mathrm{mg} / \mathrm{kg} /$ day for 6 weeks at a volume of $1 \mathrm{ml} /$ $\mathrm{kg}$ body weight.

- Duloxetine hydrochloride (Eli Lilly, Indianapolis, IN) was dissolved in distilled water and administered 
intravenously as a single dose of $1 \mathrm{mg} / \mathrm{kg}$ at a volume of $1 \mathrm{ml} / \mathrm{kg}$ body weight.

The doses of the administered agents were taken from the literature and were confirmed/adjusted in our laboratory in preliminary experiments.

\section{Study design}

After 6 weeks of 13-cis-RA administration, cystometric studies were performed to assess the effect of 13-cis-RA on the micturition cycle and to examine the influence of duloxetine on the cystometric parameters modified by 13-cis-RA treatment. They were immediately followed by behavioural tests. Blood samples were then collected and the hypothalamus and amygdala were isolated to determine the levels of 13-cis-RA and $\mathrm{CRF}$.

\section{Surgical procedures}

The surgical procedures have been previously described in detail [18]. In brief, the abdominal wall was opened with a vertical midline incision of approximately $10 \mathrm{~mm}$. A doublelumen catheter was inserted through the apex of the bladder dome and fixed with a 6-0 suture. In the same session the right femoral vein was catheterized via an inguinal approach.

\section{Conscious cystometry}

Cystometric investigations were performed 3 days after the surgical procedures and after 6 weeks of 13-cis-RA treatment. The bladder catheter was connected via a three-way stopcock to a pressure transducer (FT03) and to a microinjection pump (CMA 100). Cystometry was performed by slowly filling the bladder with physiological saline at a constant rate $0.05 \mathrm{ml} /$ min to elicit repetitive voiding. Micturition volumes were measured by means of a fluid collector attached to a force displacement transducer (FT03C). The measurements in each animal represent the average of five bladder micturition cycles after obtaining repetitive voiding. The mean values from all animals in each condition were averaged to create pooled data for each condition.

The following cystometric parameters were recorded: basal pressure $\left(\mathrm{BP}, \mathrm{cm} \mathrm{H}_{2} \mathrm{O}\right)$, threshold pressure ( $\mathrm{TP}, \mathrm{cm} \mathrm{H}_{2} \mathrm{O}$ ), micturition voiding pressure (MVP, $\mathrm{cm} \mathrm{H}_{2} \mathrm{O}$ ), voided volume (VV, ml), postvoid residual (PVR, ml), volume threshold (VT, $\mathrm{ml}$ ), voiding efficiency (VE, \%), intercontraction interval (ICI, $\mathrm{s}$ ), bladder contraction duration (BCD, s), relaxation time (RT, s), bladder compliance $\left(\mathrm{BC}, \mathrm{ml} / \mathrm{cm} \mathrm{H}_{2} \mathrm{O}\right)$, and $\mathrm{DO}$ index (DOI, $\mathrm{cm} \mathrm{H}_{2} \mathrm{O} / \mathrm{ml}$; calculated as the sum of the amplitudes of all detrusor contractions during the filling phase divided by the functional bladder capacity [17-20]), amplitude of nonvoiding contractions ( $\mathrm{ANVC}, \mathrm{cm} \mathrm{H}_{2} \mathrm{O}$ ), frequency of nonvoiding contractions (FNVC, times/filling phase), and volume threshold to elicit NVC (VTNVC, \%) [19].

\section{Locomotor activity}

The locomotor activity of animals was assessed using a Digiscan apparatus. Horizontal activity was defined as the total number of infrared light beam interruptions during $1 \mathrm{~h}$ of measurement.

\section{Forced swim test}

The immobility time was measured. The rats were considered to be immobile when they remained floating passively, performing slow motion movements to keep their head above the water.

\section{Determining the plasma level of 13-cis-retinoic acid}

The plasma levels of 13-cis-RA were measured using reversed-phase high-performance liquid chromatography. The absorbance of the samples was identified at $354 \mathrm{~nm}$ and 13-cis-RA concentrations were determined by comparison with a standard curve.

\section{Determining the hypothalamus, amygdala and plasma levels of CRF}

Using the stereotactic atlas of the rat's brain and the bregma as the point of reference, the hypothalamus and amygdala were isolated. CRF concentrations were determined using a highsensitivity commercial immunoenzymatic assay (Alpco, Salem, NH, USA) in line with the manufacturer's instructions.

\section{Statistical analysis}

The data obtained were evaluated by one-way analysis of variance followed by Tukey's post hoc test (Statistica, v. 10). All results are presented as the means \pm standard error of the mean (SEM). Values of $p<0.05$ were considered to indicate statistically significant differences.

\section{Results}

The body weights of the rats measured weekly did not show any significant differences between the animal groups examined throughout the entire experiment. Daily monitoring of the animals confirmed that they did not manifest any signs of distress as a result of the repeated injections. 
Fig. 1 Mean values of cystometric parameters (basal pressure, threshold pressure, micturition voiding pressure, voided volume, postvoid residual, and volume threshold) characteristic of detrusor overactivity induced by treatment with 13-cis-RA in the different treatment groups showing the effects of treatment with duloxetine (CON control group). a Basal pressure $(* * * p<0.001$, CON vs. 13-cis-RA). b Threshold pressure $(* * * p<0.001$, CON vs. 13-cis-RA). c Micturition voiding pressure $(* * p<0.01, \mathrm{CON}$ vs.

13-cis-RA). d Voided volume ( $* p<0.05, \mathrm{CON}$ vs. 13-cis-RA). e Postvoid residual. f Volume threshold (**p $p<0.01, \mathrm{CON}$ vs. 13-cis-RA). All results are presented as means \pm SEM ( $n=15$ rats per group). The data obtained were evaluated using one-way analysis of variance followed by Tukey's post hoc test. $* p<0.05, * * p<0.01$, $* * * p<0.001$
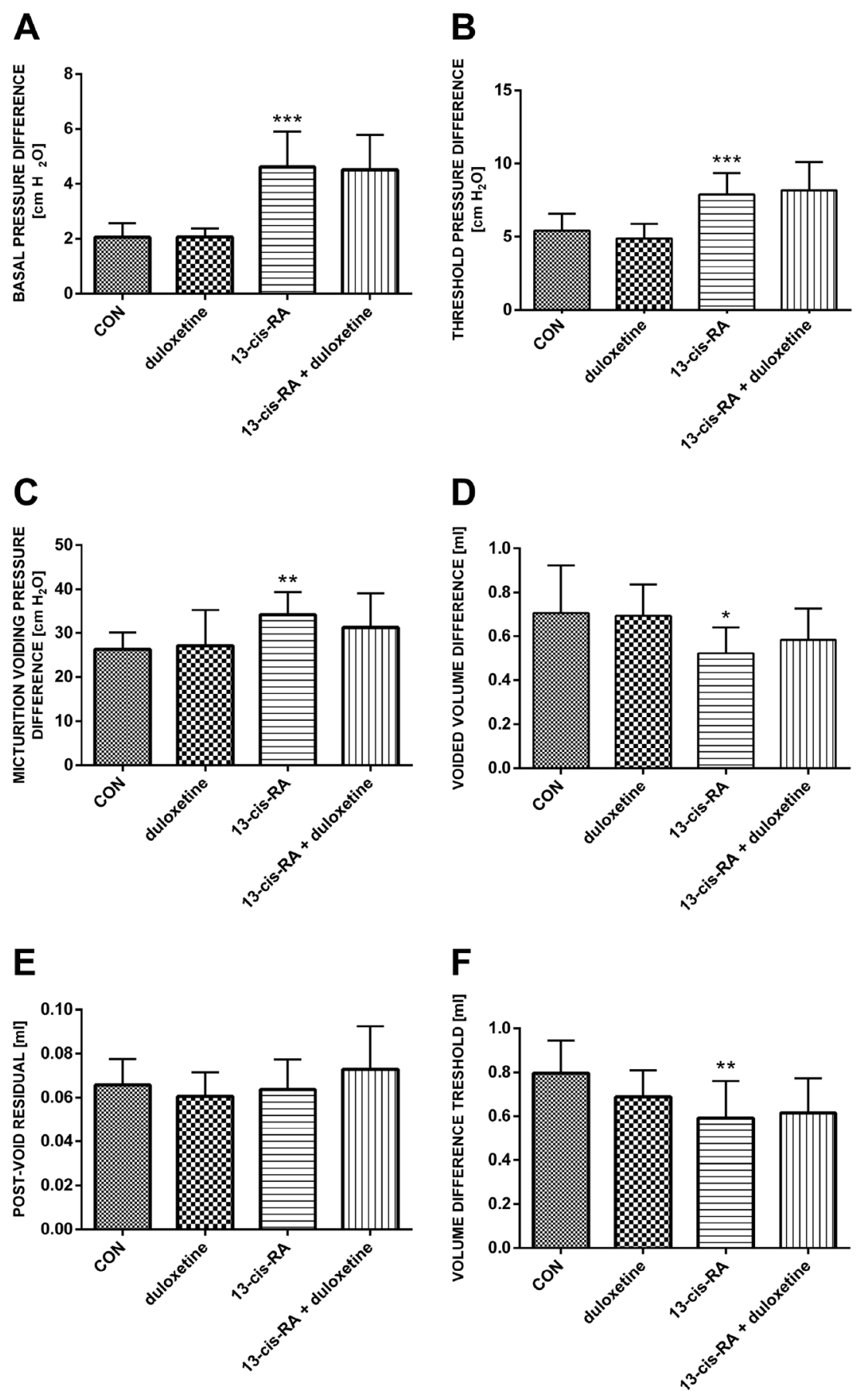

\section{Cystometric study}

Rats treated with a single dose of duloxetine did not show any significant differences in cystometric parameters compared with the control group. Treatment with 13-cis-RA led to changes in cystometric parameters characteristic of DO. Increases were observed in BP, TP, MVP, DOI, ANVC and FNVC, while decreases were observed in VV, VT, ICI, BC and VTNVC. Treatment with 13-cis-RA did not lead to any changes in PVR, VE, BCD or RT. Intravenous administration of duloxetine to animals previously treated with 13-cis-RA led to decreases in DOI, ANVC and FNVC, and to increases in $\mathrm{BC}$ and VTNVC. After administration of duloxetine, no statistically significant changes were observed in BP, TP, MVP, PVR, VT, VE, ICI, BCD or RT (Figs. 1, 2 and 3).

\section{Behavioural study}

Duloxetine administered as a single dose did not produce any statistically significant changes in the animals in the forced 
Fig. 2 Mean values of cystometric parameters (voiding efficiency, intercontraction interval, bladder contraction duration, relaxation time, bladder compliance, and detrusor overactivity index) characteristic of detrusor overactivity induced by treatment with 13-cis-RA in the different treatment groups showing the effects of treatment with duloxetine ( $\mathrm{CON}$ control group). a Voiding efficiency. b Intercontraction interval (*** $p<0.001, \mathrm{CON}$ vs. 13 -cisRA). c Bladder contraction duration. $\mathbf{d}$ Relaxation time. $\mathbf{e}$ Bladder compliance (*** $p<0.001$, CON vs. 13 -cisRA; ${ }^{\wedge} p<0.01,13$-cis-RAvs. $13-$ cis-RA + duloxetine. f Detrusor overactivity index $(* * * p<0.001$, CON vs. 13-cis-RA;

$\wedge \wedge p<0.001,13-c i s-R A$ vs. 13cis-RA + duloxetine). All results are presented as means \pm SEM ( $n=15$ rats per group). The data obtained were evaluated using one-way analysis of variance followed by Tukey's post hoc test. $\wedge^{\wedge} p<0.01, \wedge^{\wedge \wedge} p<0.001$, $* * * p<0.001$
A

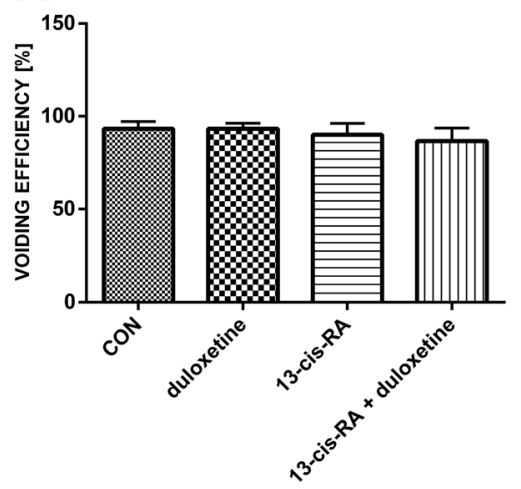

C

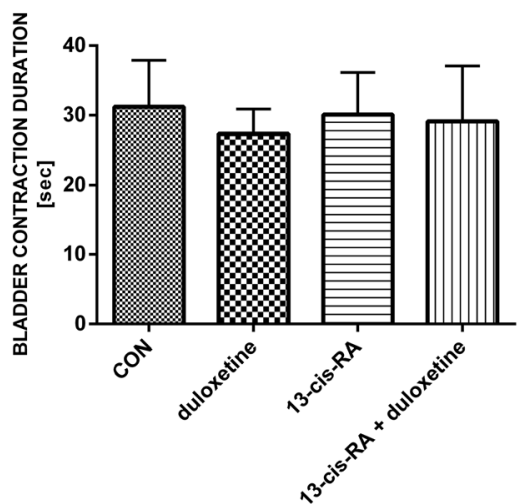

E

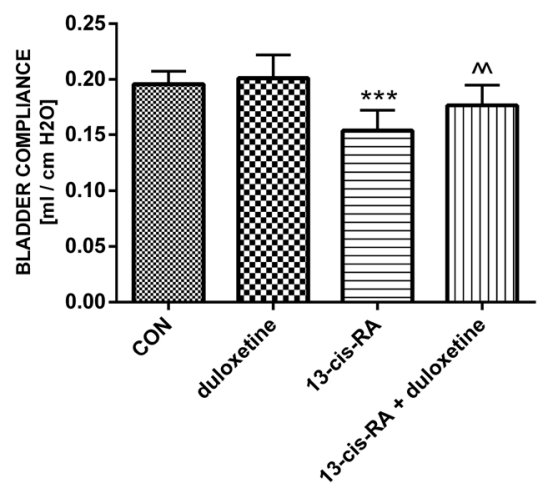

B

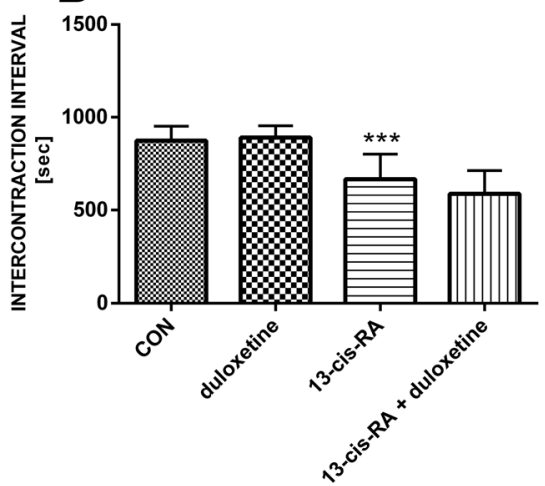

D
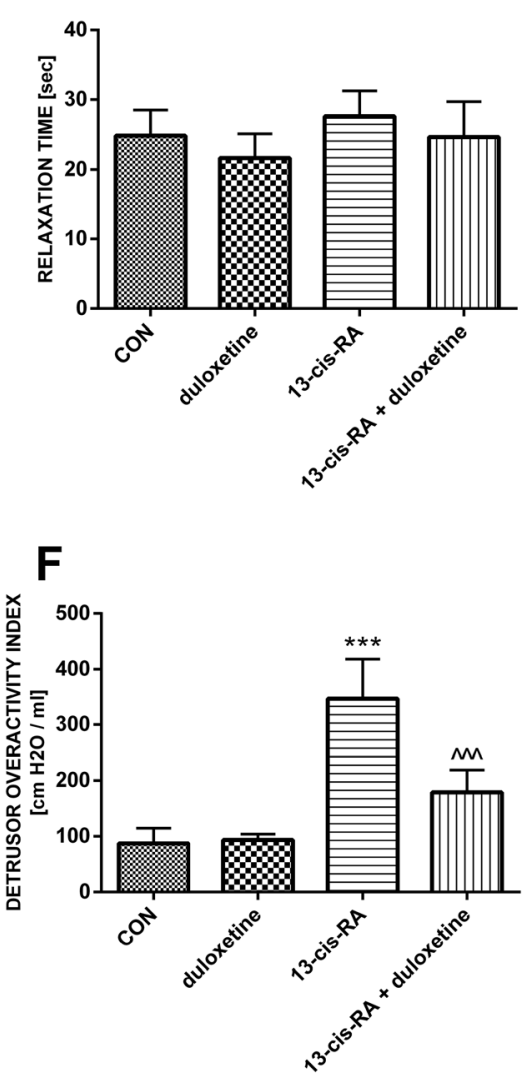

swim test. Treatment with 13-cis-RA led to an increase in the immobility time compared with the control group. Treatment with duloxetine in animals previously treated with 13-cis-RA led to a significant decrease in the immobility time to the level found in the control animals (Fig. 4). None of the treatments examined affected the locomotor activity of the animals (Fig. 5).

\section{Body weight}

No statistically significant differences in body weight were seen among the treatment groups. The mean body weights of the animals in the control group and treatment groups were $71.3 \pm 4.5 \mathrm{~g}$ and $76.1 \pm 6.1 \mathrm{~g}$, respectively, at the beginning of the experiments, and $242.6 \pm 12.4 \mathrm{~g}$ and $231.7 \pm 10.6 \mathrm{~g}$, respectively, at the end of the experiments.

\section{3-cis-Retinoic acid plasma levels}

The level of 13-cis-RA in the plasma from animals treated with 13 -cis-RA was $0.58 \pm 0.021 \mu \mathrm{g} / \mathrm{ml}$, while the plasma from animals in the control group contained essentially no measurable 13-cis-RA. 


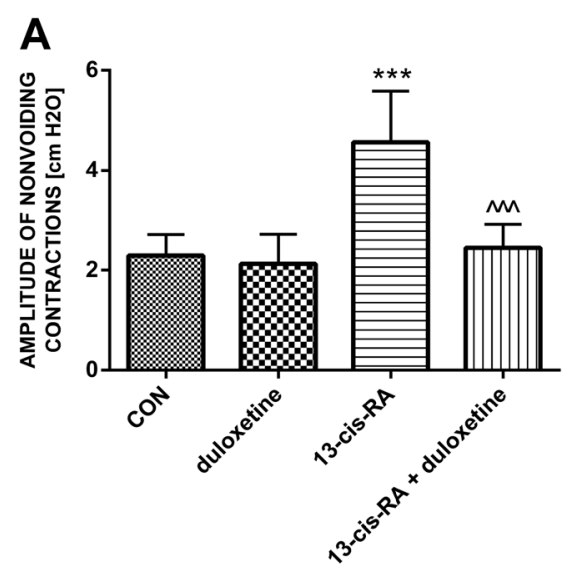

B
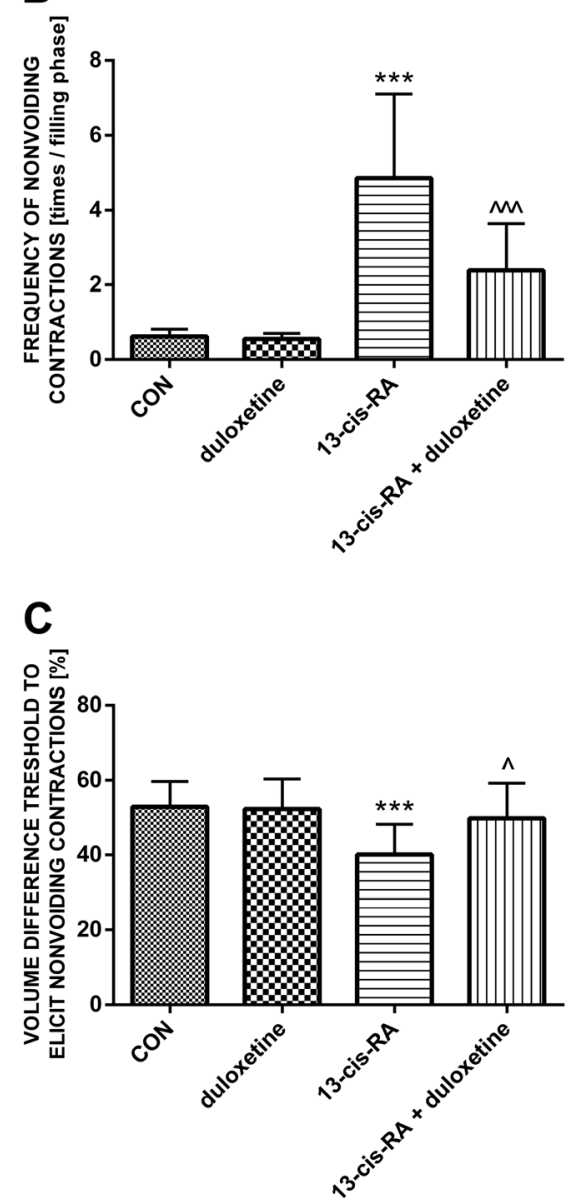

\section{CRF levels}

Treatment with a single dose of duloxetine had a statistically significant impact on CRF levels in both the examined brain structures and the plasma. The animals treated with 13-cis-RA showed increased levels of CRF in he hypothalamus, amygdala and plasma. Administration of duloxetine to rats previously treated with 13-cis-RA caused CRF levels to decrease in the hypothalamus, amygdala and plasma (Fig. 6).
Fig. 3 Mean values of cystometric parameters (amplitude of nonvoiding contractions, frequency of nonvoiding contractions, and volume threshold to elicit nonvoiding contractions) characteristic of detrusor overactivity induced by treatment with 13 -cis-RA in the different treatment groups showing the effects of treatment with duloxetine ( $C O N$ control group). a Amplitude of nonvoiding contractions $\left({ }^{* * *} p<0.001, \mathrm{CON}\right.$ vs. $13-c i s-\mathrm{RA} ;{ }^{\wedge \wedge} p<0.001,13-c i s-\mathrm{RA}$ vs. $13-$ cis-RA + duloxetine). b Frequency of nonvoiding contractions $\left(* * * p<0.001, \mathrm{CON}\right.$ vs. $13-c i s-\mathrm{RA} ;{ }^{\wedge} \wedge p<0.001,13-c i s-\mathrm{RA}$ vs. $13-$ cis-RA + duloxetine). c Volume threshold to elicit nonvoiding contractions $\left(* * * p<0.001, \mathrm{CON}\right.$ vs. $13-c i s-\mathrm{RA} ;{ }^{\wedge} p<0.05,13-c i s-\mathrm{RA}$ vs. 13-cis-RA + duloxetine). All results are presented as means \pm SEM ( $n=15$ rats per group). The data obtained were evaluated using one-way analysis of variance followed by Tukey's post hoc test. $\wedge^{\wedge} p<0.05$, $\wedge^{\wedge \wedge} p<0.001, * * * p<0.001$

\section{Discussion}

The model of depression induced by 13-cis-RA, which has also been shown to cause DO symptoms, was used in this study. Clinical trials have shown that therapeutic doses of 13-cis-RA administered to patients with severe acne can induce depressive behaviour [21]. These findings were confirmed in preclinical studies in which long-term administration of 13-cis-RA led to increases in immobility time in the forced swim test, which is consistent with increased depression-related behaviour [22]. In the experiments presented here, the rats received a dose identical to that used in acne treatment, and the determined concentrations of 13-cis-RA in plasma were analogous to those found in humans [23].

Treatment with duloxetine was found to have an antidepressant effect, as shown by significant decreases in the immobility time to the times observed in the control animals, with a simultaneous lack of effect on their locomotor activity. Treatment with duloxetine led to significant decreases in DOI, ANVC and FNVC and to increases in BC and VTNVC. Duloxetine appeared to have no effects on the other analysed parameters, namely BP, TP, MVP, PVR, VT, VE, ICI, BCD and RT. The demonstrated decrease in DOI seems to be very

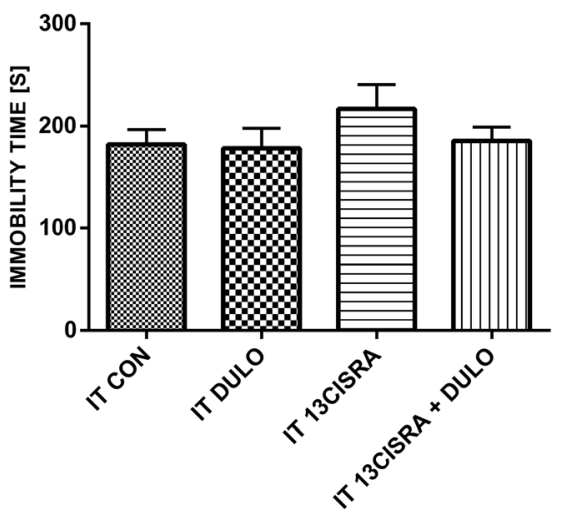

Fig. 4 Immobility times in rats in the different treatment groups. All results are presented as means $\pm \operatorname{SEM}(n=15$ rats per group). The data obtained were evaluated using one-way analysis of variance (ANOVA) followed by Tukey's post hoc test 


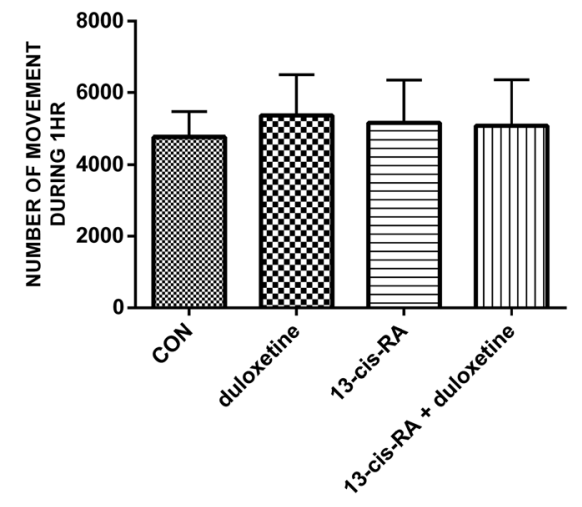

Fig. 5 locomotor activity (number of movements) in rats in the different treatment groups. All results are presented as the means $\pm \operatorname{SEM}(n=15$ rats per group). The data obtained were evaluated using one-way analysis of variance followed by Tukey's post hoc test

important, considering that an increase in this parameter is a characteristic feature of DO that allows its intensity to be determined. It is believed that DOI reflects the contraction activity of the bladder with far greater precision than ANVC, FNVC, MVP, ICI, BP or BC $[18,19]$. The observed increase in VTNVC following administration of duloxetine is very interesting, because this parameter is considered a preclinical equivalent to the volume at the first involuntary detrusor contraction measured during urodynamic investigations in humans [24]. The dose used in this study was the minimally effective dose, which showed antidepressant activity in the forced swim test and simultaneously affected the cystometric parameters DOI, ANVC, FNVC, BC and VTNVC. This might suggest that duloxetine could be used in the pharmacotherapy of $\mathrm{OAB}$ dry, especially in patients with accompanying depression. It cannot be ruled out that higher doses administered for a longer period could effectively treat $\mathrm{OAB}$ wet.

Recently published studies on the link between $\mathrm{OAB}$ and depression were population-based epidemiological surveys and yielded conflicting results $[4,25]$. In a study by Lai et al. [26], 27.5\% of OAB patients had depression as confirmed by the Hospital Anxiety and Depression Scale (HADS $\geq 8$ ), and affected individuals reported significantly higher HADS-D depression scores than age-matched controls. No standards for the treatment of patients with DO and accompanying depression have yet been developed, because $\mathrm{OAB}$ is a syndrome and does not exist as an entity. So far only one case report has been published in which the authors confirmed the efficacy of duloxetine in a female adolescent with $\mathrm{OAB}$ and depression [14]. It is worth noting that the patient showed resistance to antimuscarinic drugs and standard antidepressants.

The rationale for the use of duloxetine in DO stems from the direct inhibition of sensory afferents by the serotonergic system, which results in a reduction in bladder detrusor activity and a modulating effect of serotonin and noradrenaline on the micturition centres in the sacral spinal cord [27]. It has
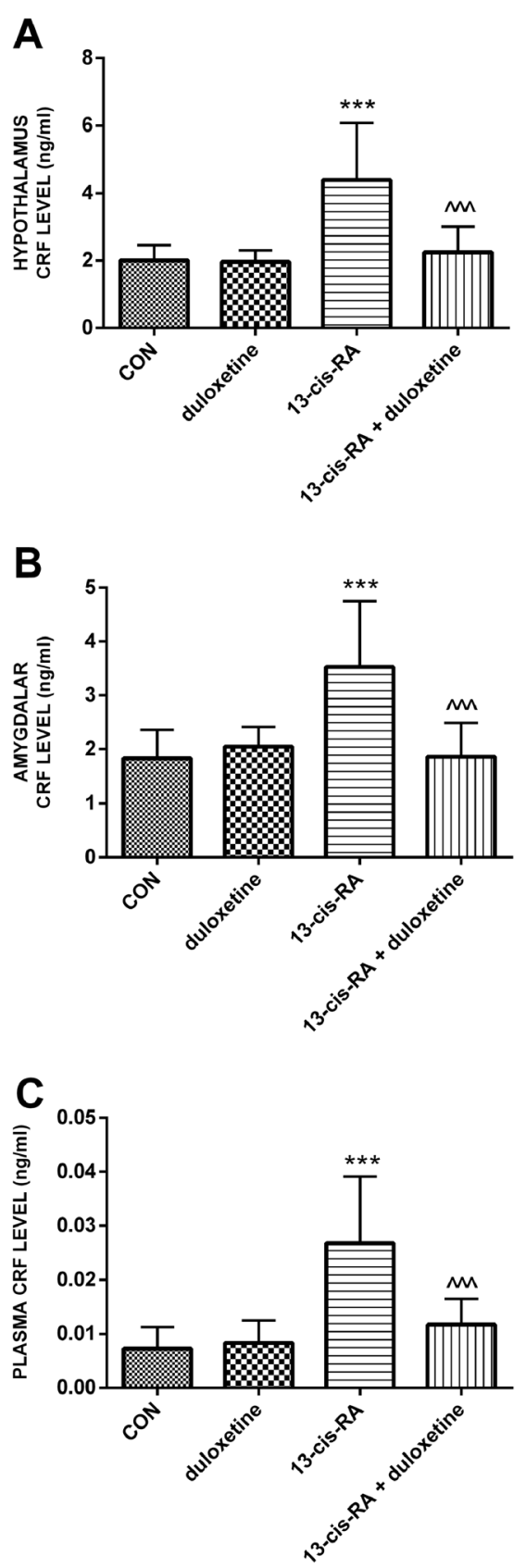

Fig. 6 Mean CRF levels in the hypothalamus, amygdala and plasma of rats in the different treatment groups (CON control group). a Hypothalamus $\left(* * * p<0.001\right.$, CON vs. $13-c i s-\mathrm{RA} ;{ }^{\wedge \wedge} p<0.001,13-$ cis-RA vs. 13-cis-RA + duloxetine). b Amygdala (*** $p<0.001$, CON vs. 13-cis-RA; ${ }^{\wedge \wedge} p<0.001,13$-cis-RA vs. 13-cis-RA + duloxetine). c Plasma $\left(* * * p<0.001\right.$, CON vs. $13-c i s-R A ;{ }^{\wedge} \wedge p<0.001,13-c i s-R A$ vs. 13-cis-RA + duloxetine). All results are presented as means \pm SEM ( $n=15$ rats per group). The data obtained were evaluated using one-way analysis of variance followed by Tukey's post hoc test. ${ }^{\wedge} \wedge p<0.001$, $* * * p<0.001$

been shown that acute administration of antidepressants increases the threshold for the spinal voiding reflex, while chronic administration affects the CNS-related components of the micturition reflex, which suggests that duloxetine could 
inhibit bladder overactivity in patients with coexisting depression [15]. There are reports that SNRIs can induce urine retention, but this was not confirmed in our study. Duloxetine was found to have no effect on PVR.

It ha been demonstrated that duloxetine increases bladder capacity and inhibits DO induced by acetic acid, and its effects are reversed by administration of a nonselective 5-HT receptor antagonist [28]. The fact that DO induced by chemical irritation is mediated at least in part by a spinal reflex activated by nociceptive $\mathrm{C}$-fibre afferents of the urinary bladder suggests the possibility that duloxetine affects not only supraspinal micturition control mechanisms but also spinal reflexes.

Clinical trials have shown that duloxetine reduces the number of frequency and urgency episodes, and improves quality of life scores, while not changing the mean voided volume $[15,16]$. It has not been confirmed that it has any effects on heart rate, blood pressure or arrhythmogenic tendencies on EEG, which is significant because the majority of patients with depression and OAB are elderly. Duloxetine was found to have greater efficacy than placebo in patients with $\mathrm{OAB}$ due to either DO or sensory urgency, and also in patients with MUI $[16,29]$. Due to the drug's clinically proven effectiveness in the treatment of depression, it has been suggested that it should be the first-choice treatment in patients with depression and accompanying $\mathrm{OAB}[15]$.

In this study duloxetine was found to lower CRF levels in the hypothalamus, amygdala and plasma that were previously increased by administration of 13-cis-RA. CRF hypersecretion seems to be a common element in the aetiopathogenesis of depression and $\mathrm{OAB}$ stemming from disturbances to the hypothalamic-pituitary-adrenal axis. There is growing evidence to support the considerable impact of CRF on urinary bladder function. This neurohormone has been found to decrease the threshold of afferent impulsation of the micturition reflex and to increase bladder detrusor contraction activity. CRF also plays an important role in micturition control mechanisms at the level of the spinal cord [30]. Preclinical studies have shown that selective $\mathrm{CRF}_{1}$ antagonists reduce DO symptoms [10]. It has been shown that selective destruction of afferent $\mathrm{C}$-fibres leads to decreased expression of CRF, which indicates that this neurohormone is important for conducting the afferent impulsation from the urinary bladder [31]. Increased CRF expression has also been found in the urothelium and suburothelial ganglions in an animal model of cystitis.

It has been shown that brain regions characterized by a high density of $\mathrm{CRF}_{1}$ receptors (such as the hippocampus, the prefrontal cortex and Barrington's nucleus in the brainstem amygdala), apart from their well-known role in the body's response to stress factors, are also involved in regulation of the micturition reflex, and this suggests that antagonists to this receptor might be an interesting target for $\mathrm{OAB}$ pharmacotherapy, especially in patients with accompanying depression
[32]. Another CNS structure found to be a common element in the aetiopathogenesis of $\mathrm{OAB}$ and depression is the anterior cingulate cortex, whose increased activation has been detected on MRI in patients with OAB and patients with depression. SPECT also shows features of hypoperfusion and hypometabolism of this structure in both these groups of patients.

\section{Conclusions}

This study provides further data concerning the links between the aetiopathogenesis of $\mathrm{OAB}$ and depression. Three main findings concerning the action of duloxetine should be particularly underlined: (1) it reduces the cystometric changes of DO induced by 13-cis-RA administration, (2) it shows antidepressant activity, and (3) it decreases CRF levels in the hypothalamus, amygdala and plasma. This is the first study showing the efficacy of duloxetine in an animal model of DO induced by depression. Further studies in patients with DO and coexisting depression are warranted to confirm these experimental results.

\section{Compliance with ethical standards}

Funding This study was supported by Funds for Statutory Activity of the Medical University of Lublin, Poland.

\section{Conflicts of interest None.}

Open Access This article is distributed under the terms of the Creative Commons Attribution 4.0 International License (http:// creativecommons.org/licenses/by/4.0/), which permits unrestricted use, distribution, and reproduction in any medium, provided you give appropriate credit to the original author(s) and the source, provide a link to the Creative Commons license, and indicate if changes were made.

\section{References}

1. Engel WJ. Uropsychiatry. J Mich State Med Soc. 1964;63:273-7.

2. Steers WD, Lee KS. Depression and incontinence. World J Urol. 2001;19(5):351-7.

3. Vrijens D, Drossaerts J, van Koeveringe G, et al. Affective symptoms and the overactive bladder - a systematic review. J Psychosom Res. 2015;78:95-108.

4. Melville JL, Walker E, Katon W, et al. Prevalence of comorbid psychiatric illness and its impact on symptom perception, quality of life, and functional status in women with urinary incontinence. Am J Obstet Gynecol. 2002;187:80-7.

5. Sung VW, West DS, Hernandez AL, et al. Association between urinary incontinence and depressive symptoms in overweight and obese women. Am J Obstet Gynecol. 2009;200:557.e1-e5.

6. Dugan E, Cohen SJ, Bland DR, et al. The association of depressive symptoms and urinary incontinence among older adults. J Am Geriatr Soc. 2000;48(4):413-6.

7. Hirayama A, Torimoto K, Mastusita C, et al. Risk factors for newonset overactive bladder in older subjects: results of the Fujiwarakyo study. Urology. 2012;80(1):71-6. 
8. Bale TL, Vale WW. CRF and CRF receptors: role in stress responsivity and other behaviors. Annu Rev Pharmacol Toxicol. 2004;44:525-57.

9. Yankelevitch-Yahav R, Franko M, Huly A, et al. The forced swim test as a model of depressive-like behavior. J Vis Exp. 2015;2(97). doi:10.3791/52587.

10. Klausner AP, Streng T, Na YG, et al. The role of corticotropin releasing factor and its antagonist, astressin, on micturition in the rat. Auton Neurosci. 2005;123:26-35.

11. Klausner A. Corticotrophin releasing factor reduce the micturition threshold when administered at central, spinal and peripheral levels. J Urol. 2004;171:453-9.

12. Raskin J, Goldstein D, Mallinckrodt C, et al. Duloxetine in the long term treatment of major depressive disorder. J Clin Psychiatry. 2003;64:1237-44.

13. Jost WH, Marsalek P. Duloxetine in the treatment of stress urinary incontinence. Ther Clin Risk Manag. 2005;1:259-64.

14. Wang SM, Lee HK, Kweon YS, et al. Overactive bladder successfully treated with duloxetine in a female adolescent. Clin Psychopharmacol Neurosci. 2015;13(2):212-4.

15. Di Rezze S, Frasca V, Inghilleri M, et al. Duloxetine for the treatment of overactive bladder syndrome in multiple sclerosis: a pilot study. Clin Neuropharmacol. 2012;35(5):231-4.

16. Steers WD, Herschorn S, Kreder KJ, et al. Duloxetine compared with placebo for treating women with symptoms of overactive bladder. BJU Int. 2007;100:337-45.

17. Wróbel A, Rechberger T. An animal model of detrusor overactivity induced by depression. J Pharmacol Toxicol Methods. 2016;80:1925 .

18. Wróbel A, Rechberger T. The influence of rho-kinase inhibition on acetic acid-induced detrusor overactivity. Neurourol Urodyn. 2017;36:263-70.

19. Wróbel A, Rechberger T. The effect of combined treatment with a B3 AR agonist and a ROCK inhibitor on detrusor overactivity. Neurourol Urodyn. 2017;36:580-8.

20. Wróbel A, Serefko A, Poleszak E, et al. Fourteen-day administration of corticosterone may induce detrusor overactivity symptoms. Int Urogynecol J. 2016;27(11):1713-21.
21. Bremner JD, McCaffery P. The neurobiology of retinoic acid in affective disorders. Prog Neuropsychopharmacol Biol Psychiatry. 2008;32:315-31.

22. Cai L, Li R, Zhou JN. Chronic all-trans retinoic acid administration induces CRF over-expression accompanied by AVP up-regulation and multiple CRF-controlling receptors disturbance in the hypothalamus of rats. Brain. 2015;1601:1-7.

23. Kerr IG, Lippman ME, Jenkins J, et al. Pharmacology of 13-cisretinoic acid in humans. Cancer Res. 1982;42:2069-73.

24. Behr-Roussel D, Oger S, Pignol B, et al. Minimal effective dose of dysport and botox in a rat model of neurogenic detrusor overactivity. Eur Urol. 2012;61:1054-61.

25. Zorn BH, Montgomery H, Pieper K, et al. Urinary incontinence and depression. J Urol. 1999;162:82-4.

26. Lai HH, Shen B, Rawal A, et al. The relationship between depression and overactive bladder/urinary incontinence symptoms in the clinical OAB population. BMC Urol. 2016;16:60.

27. Burgard EC, Fraser MO, Thor KB. Serotonergic modulation of bladder afferent pathways. Urology. 2003;62:10-5.

28. Katofiasc MA, Nissen J, Audia JE, et al. Comparison of the effects of serotonin selective, norepinephrine selective, and dual serotonin and norepinephrine reuptake inhibitors on lower urinary tract function in cats. Life Sci. 2002;71:1227-36.

29. Bump R, Norton P, Zinner N, et al. Mixed urinary incontinence symptoms: urodynamic findings, incontinence severity, and treatment response. Obstet Gynecol. 2003;102:76-83.

30. Vincent SR, Satoh K. Corticotropin-releasing factor (CRF) immunoreactivity in the dorsolateral pontine tegmentum: further studies on the micturition reflex system. Brain Res. 1984;308(2):387-91.

31. Skofitsch G, Zamir N, Helke CJ, et al. Corticotropin releasing factor-like immunoreactivity in sensory ganglia and capsaicin sensitive neurons of the rat central nervous system: colocalization with other neuropeptides. Peptides. 1985;6(2):307-18.

32. Imaki T, Nahan JL, Rivier C, et al. Differential regulation of corticotropin-releasing factor mRNA in rat brain regions by glucocorticoids and stress. J Neurosci. 1991;11(3):585-99. 